\title{
Future XMASS project
}

\section{Ko Abe ${ }^{1}$, for the XMASS collaboration}

Kamioka Observatory, Institute for Cosmic Ray Research, University of Tokyo

Higashi-Mozumi 456, Kamioka-cyo, Hida-city, Gifu 506-1205, Japan

E-mail: abe@suketto.icrr.u-tokyo.ac.jp

XMASS is a single phase liquid xenon detector. It is designed for a program of physics targets. The present XMASS-I detector targets direct dark matter detection. Background rejection is based on position information. Due to strong stopping power of xenon, beta and gamma rays from the outside cannot penetrate deep into the liquid xenon. By eliminating event interacting outer region, most background can be eliminated.

By using the data obtained by XMASS-I, dark matter, axion and double electron capture searches were carried out and the results were published.

XMASS-1.5 is next step of XMASS project to perform dark matter search with higher sensitivity. Detector design is now ongoing to improve sensitivity below $10^{-46} \mathrm{~cm}^{2}$ cross section for $100 \mathrm{GeV}$ WIMP by increasing total amount of liquid xenon and reducing background level below $10^{-5}$ $/ \mathrm{day} / \mathrm{kg} / \mathrm{keVee}$.

PMT exchange for surface background reduction is one of the largest modification from XMASSI. New PMT R13111 has dome-shaped photocathode which is very effective for identification of surface background. Confirmation of performances including sensitivity of side part of photocathode is already done. Radioactivity reduction of R13111 is ongoing by screening all parts. Up to the present, reductions of $\sim 1 / 3$ for $U$ chain $\left({ }^{226} \mathrm{Ra}\right)$ and Th chain, $<1 / 8$ for ${ }^{40} \mathrm{~K}$ and $\sim 1 / 6$ for ${ }^{60}$ Co from XMASS-I PMT are achieved.

38th International Conference on High Energy Physics

3-10 August 2016

Chicago, USA

\section{${ }^{1}$ Speaker}




\section{Introduction}

XMASS [1] is a single phase liquid xenon scintillation detector. It is designed to achieve a range of physics goals as the program develops: dark matter, neutrinoless double beta decay and ${ }^{7} \mathrm{Be} / \mathrm{pp}$ solar neutrinos. The present XMASS-I detector is the first step in the project and targets direct dark matter detection with $832 \mathrm{~kg}$ of liquid xenon in its sensitive volume. The experiment is located in the Kamioka Mine under Mt. Ikenoyama in Japan, which provides $2700 \mathrm{~m}$ water equivalent in shielding from cosmic rays.

\subsection{XMASS-I detector}

Figure 1 shows the XMASS-I detector. The sensitive region of XMASS-I, an approximation to a sphere of about 80 $\mathrm{cm}$ diameter, is surrounded by 642 PMTs. In order to reduce external gammas, neutrons, and cosmic-ray muon induced backgrounds, the detector is placed at the center of a water tank. The tank is $10 \mathrm{~m}$ in diameter and $10 \mathrm{~m}$ high. 72 20-inch PMTs (Hamamatsu R3600) inside the water tank provide an active muon veto. Further background rejection is done by usign event position information. Due to the strong stopping power of xenon, beta and gamma rays from the outside cannot penetrate deep into the liquid xenon. By eliminating events interacting in the outer region, most of the background can be eliminated.

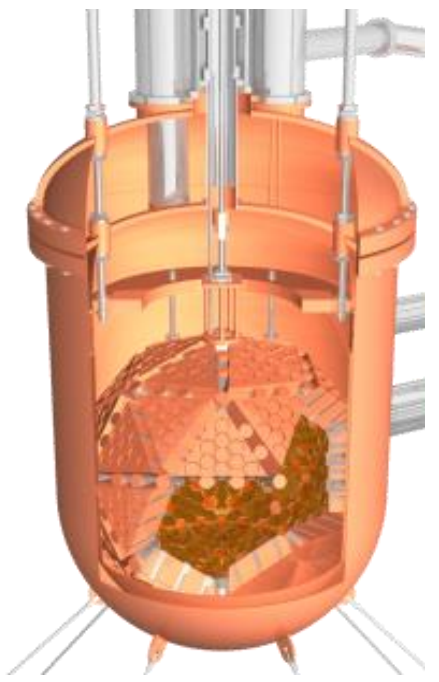

Figure 1: XMASS-I detector More details are described in Ref. [2]. By using the data obtained by XMASS-I, dark matter, axion and double electron capture searches were carried out and the results were published [3-8].

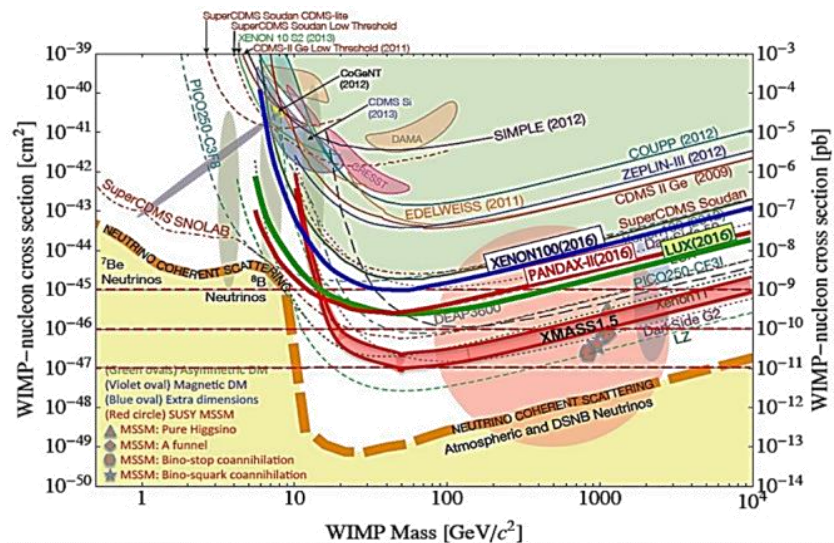

Figure 2: Expected sensitivity for WIMP dark matter.

\section{XMASS-1.5, Next step of XMASS project}

XMASS-1.5 is next step of XMASS project to perform dark matter search with higher sensitivity. Detector design is now ongoing to realize sensitivity below $10^{-46} \mathrm{~cm}^{2}$ cross section for $100 \mathrm{GeV}$ WIMP (figure 2) by increasing total amount of liquid xenon to 5 tons and reducing background level below $10^{-5} / \mathrm{day} / \mathrm{kg} / \mathrm{keVee}$.

One of the largest modification from XMASS-I is PMT. Photocathode shape of PMT is changed from flat to dome-shape. Surface events which is one of the largest background can be identified very effectively by this domeshape photocathode as shown in figure 3. R13111 is newly developed 3-inch dome-shaped photocathode PMT for XMASS-1.5. Performance check was already done for the first batch. Besides basic properties such as low dark rate and stable operation inside liquid xenon, sensitivity of side part of photocathode which is most important for surface background reduction is confirmed as designed level, larger than $80 \%$ of sensitivity at dome top. Radioactivity reduction 


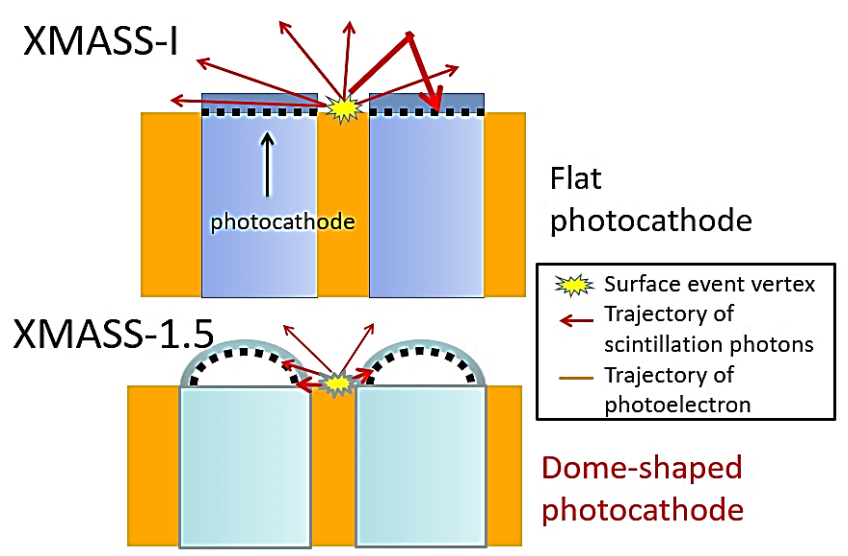

Figure 2: PMTs used in XMASS-I and XMASS-1.5

for PMT is ongoing by screening all parts. 1/10 of R10789 used in XMASSI is set as target value to realize $10^{-5}$ $/$ day $/ \mathrm{kg} / \mathrm{keV}_{\text {ee }}$ level of background. Figure 4 shows achieved radioactivity for R13111 comparing to R10789. Current achieved reduction from $\mathrm{R} 10789$ is $\sim 1 / 3$ for $\mathrm{U} / \mathrm{Th}$ chain, $<1 / 8$ for ${ }^{40} \mathrm{~K}$ and $\sim 1 / 6$ for ${ }^{60} \mathrm{Co}$.

\section{Summary}

XMASS is a single phase liquid xenon detector. XMASS-1.5 is next step of XMASS project to achieve sensitivity below $10^{-}$ ${ }^{46} \mathrm{~cm}^{2}$ cross section for $100 \mathrm{GeV}$ WIMP dark matter. PMT exchange for surface background reduction is one of ther largest modification from XMASS-I. Dome-shaped photocathode of new PMT R13111 is very effective for indentification of surface backgroud. Confirmation of performance including sensitivity of side part of photocathode was already done. Ongoing radioactivity reduction of $\mathrm{R} 13111$ achieved $\sim 1 / 3$ for $\mathrm{U}$ chain $\left({ }^{226} \mathrm{Ra}\right)$ and $\mathrm{Th}$ chain, $<1 / 8$ for ${ }^{40} \mathrm{~K}$ and $\sim 1 / 6$ for ${ }^{60} \mathrm{Co}$ from XMASS-I PMT up to the present.

\section{References}

[1] Y. Suzuki et al., Low Energy Solar Neutrino Detection by using Liquid Xenon, [hep-ph/0008296]

[2] K. Abe et al., XMASS detector, Nucl. Instr. And Meth. In Phys. Res. A716 (2013) 78

[3] K. Abe et al., Light WIMP search in XMASS, Phys. Lett. B719 (2013) 78

[4] K. Abe et al., Search for solar axions in XMASS, a large liquid-xenon detector, Phys. Lett. B724 (2013) 46

[5] K. Abe et al., Search for inelastic WIMP nucleus scattering on 129Xe in data from the XMASS-I experiment, status and results, Prog. Theor. Exp. Phys. (2014) 063C01

[6] K. Abe et al., Search for Bosonic Superweakly Interacting Massive Dark Matter Particles with the XMASS-I Detector, Phys. Rev. Lett. 114 (2014) 121301

[7] K. Abe et al., Search for two-neutrino double electron capture on 124Xe with the XMASS-I detector, Phys. Lett. B759 (2016) 64, [1510.00754[nucl-ex]]

[8] K. Abe et al., Direct dark matter search by annual modulation in XMASS-I, Phys. Lett. B759 (2016) 272, [1511.04807[astro-ph.CO]] 